\title{
Effects of a 12-month educational intervention on outpatient clinicians' attitudes and behaviors concerning spiritual practices with patients
}

This article was published in the following Dove Press journal:

Advances in Medical Education and Practice

3 February 2017

Number of times this article has been viewed

\author{
Harold G Koenigl-4 \\ Kathleen Perno 5 \\ Ted Hamilton ${ }^{5}$ \\ 'Department of Psychiatry, \\ ${ }^{2}$ Department of Medicine, Duke \\ University Medical Center, Durham, \\ NC, USA; ${ }^{3}$ Department of Medicine, \\ King Abdulaziz University, Jeddah, \\ Saudi Arabia; ${ }^{4}$ School of Public Health, \\ Ningxia Medical University, Yinchuan, \\ People's Republic of China; ${ }^{5}$ Medical \\ Mission Integration, Adventist Health \\ System, Orlando, FL, USA
}

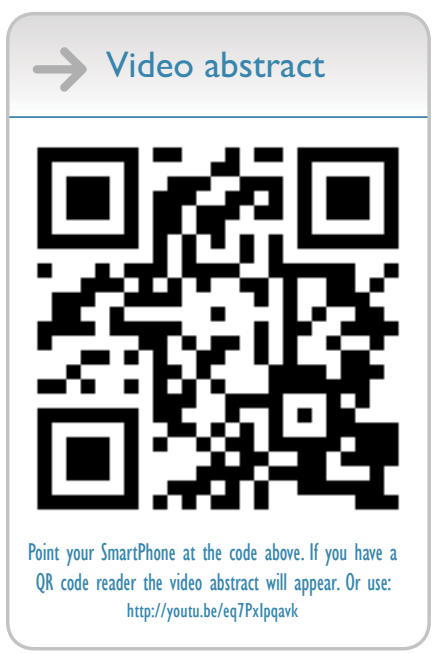

Correspondence: Harold G Koenig Duke University Medical Center, PO Box 3400, Durham, NC 27710, USA Email Harold.Koenig@duke.edu
Objective: We report here the impact of an educational training program on attitudes and practices of physicians (MDs) and mid-level practitioners (MLPs) toward controversial spiritual practices, such as practitioner-led prayer, sharing personal religious beliefs, and encouraging patients' religious beliefs. Methods: In this single-group experimental study, 427 physicians and 93 MLPs affiliated with the Adventist Health System agreed to complete a questionnaire assessing demographics, practice characteristics, religiosity, and attitudes and behaviors at baseline, 1 month, and 12 months. Changes in attitudes and practices over time were examined and baseline predictors were identified using mixed-effects regression models.

Results: For the most part, attitudes regarding praying with patients, sharing faith with patients, and encouraging patients' own religious faith did not change much during the 12-month educational training program. However, significant increases were found in frequency of praying with patients (MDs and MLPs), willingness to pray with patients (MDs), sharing their faith with patients (MDs), and encouraging patient's own religious faith (MDs and MLPs). Among physicians, predictors of praying with patients across time were older age, Christian affiliation, and importance of religion, and among MLPs, they were older age, non-White race, and importance of religion. No interaction between time and religiosity was found.

Conclusion: Although attitudes toward these mostly controversial practices were largely unaffected, the frequency of praying with patients, sharing faith, and supporting patient's own religious faith increased over time in both religious and nonreligious clinicians. Educational programs of this type may be important in changing clinicians' behaviors regarding appropriate and sensitive engagement in such activities with patients.

Keywords: physicians, health professionals, behavior, spirituality, religion, prayer

\section{Background}

There is increasing research demonstrating a link between religion/spirituality and both mental and physical health, ${ }^{1-5}$ the frequent spiritual needs present among patients with medical illness, ${ }^{6,7}$ the cost of unmet spiritual needs, ${ }^{8}$ and the requirements by accrediting bodies that clinicians respect patients' values, beliefs, and preferences (often religious or spiritual in nature). ${ }^{9}$ However, primary providers seldom address the spiritual needs of medical patients or engage in health-related spiritual activities with them - especially in outpatient settings, where there is increasingly little time to even do a standard medical evaluation. ${ }^{10}$ When clinicians do take time to engage in spiritual practices with patients, they often do so inconsistently and without clear knowledge about appropriate boundaries, and of particular concern, this activity may be driven by personal religious beliefs (rather than by the needs of the patient). For this reason, 
the training of health professionals (HPs) on how to integrate spirituality into patient care has been strongly encouraged across the health professions. ${ }^{11-15}$ In fact, a recent study of the attitudes and practices of oncology specialists toward providing spiritual care to patients concluded that training to assess and address patients' spiritual needs is "the critical next step" to meet national care quality standards in health care. ${ }^{16}$

"Faith-based" health systems have as one of their primary missions the integration of spirituality into patients' care. This is one of the most important factors that distinguish these health care systems from health care organizations that are secular in nature. To carry out this mission, and address the problem of inadequate training of HPs on how to integrate spirituality into the care of patients in outpatient settings, the Adventist Health System (AHS) - the largest Protestant health care system in the $\mathrm{USA}^{17}$ - in collaboration with Duke University decided to develop and assess the effects of a 12-month intensive educational training program (ETP) directed at primary providers. This report examines the effects of this ETP on changes in the attitudes and practices of physicians and mid-level practitioners (MLPs) toward more controversial practices such as praying with patients, sharing personal religious beliefs with patients, and encouraging patients' own religious beliefs for health reasons.

\section{Hypotheses Hypothesis I}

Given that most participants had not received training on how to integrate spirituality into patient care and that the ETP was designed specifically to provide that training, we hypothesized that the ETP would increase providers' belief that: 1) prayer with patients is appropriate under some circumstances, 2) prayer with patients is appropriate only if the patient initiates, 3) clinicians should support/encourage patients' own religious beliefs, 4) providers should not initiate prayer with patients, and 5) providers should not share their own religious beliefs with patients unless asked.

\section{Hypothesis 2}

As the ETP trained participants on when and how to engage in spiritual practices with patients and gave them explicit permission to do so, we hypothesized that the ETP would increase the frequency of praying with patients, encouraging patients' own religious faith, willingness to pray with patients, willingness to support patients' own faith, and frequency of chaplain referrals.

\section{Hypothesis 3}

Clinicians who are themselves religious might be more sensitive to the spiritual needs of patients and more comfortable engaging in spiritual activities with patients; those who had received prior training might experience greater comfort engaging in spiritual activities with patients; and a particular focus of family physicians is addressing the whole-person psychosocial needs of patients. We therefore hypothesized that attitudes toward engaging in spiritual activities with patients would be more positive and behaviors in this regard more frequent across time in clinicians who were more religious, those who had received training on spirituality during their professional education, and those in family medicine.

\section{Hypothesis 4}

Because religious clinicians might be more receptive to the permission, support, and encouragement offered during the ETP to appropriately engage in spiritual activities with patients, we hypothesized that change in such behaviors would be greater over time among providers who were more religious (ie, there would be a greater increase over time in spiritual practices among clinicians who were more religious compared to those who were less).

\section{Methods}

The design was a single-group experimental study involving clinicians in outpatient practices affiliated with the AHS in southeastern and mid-western USA ( $\sim 50 \%$ of all AHS hospitals, $73 \%$ of participants with no exposure to the topic). Between February and August 2015, a convenience sample of 1,082 physicians and MLPs (nurse practitioners or physician's assistants) were asked to participate in a 12-month ETP and research study. Of those approached, 520 (48\%) (427 physicians and 93 MLPs) in 220 practices agreed to participate in the training program and complete a questionnaire at baseline, 1 month, and 12 months during the program. The proportion of physicians and MLPs involved in the study was representative of their distribution in the practices that agreed to participate (ie, reflecting their representation in the workforce). Informed consent was obtained from all participants, and the study was approved by the institutional review boards (IRBs) of Duke University (Pro00054912) and AHS. The IRBs did not require that written informed consent be obtained from participants. Since this was an educational intervention that was entirely voluntary and involved a nonvulnerable population, the IRBs decided verbal consent was adequate and the authors were only required to provide a copy of the consent to each participant.

\section{Training program}

The ETP involved 1) participating in an orientation meeting with a Regional Faith Coordinator (RFC) hired and trained by 
AHS, 2) watching a 5-minute introductory video that further explained the rationale for the program, 3) viewing one or more of five 45 -minute educational videos ${ }^{18}$ that described how to integrate spirituality (three for primary providers, one for nurses, one for the overall team including chaplains), and 4) meeting monthly with the RFC who trained, supported, and encouraged providers and their staff on how to assess and address patients' spiritual needs. Nine full-time RFCs and a full-time RFC coordinator provided training during the 12-month program.

Given that this was a research study and participation was voluntary, not all clinicians were exposed to all four components listed above. Of those who responded at the 12-month assessment to a question that was asked about the components of the program completed $(n=427), 96 \%$ indicated that they attended the orientation meeting with RFC, $82 \%$ watched the short introductory video, $52 \%$ on average met monthly with RFCs, and $60 \%$ viewed one or more continuing medical education (CME) videos, listened to an audio $\mathrm{CD}$ of the videos, read transcripts of the videos, or attended a 2-3-hour lecture by investigators.

The training program, summarized in the CME videos, described the rationale for integrating spirituality into patient care based on current research, discussed appropriate behaviors that clinicians could be involved in with patients, and described boundaries that should not be crossed. Besides taking a spiritual history (effects of the ETP reported elsewhere $^{19}$ ), providers were encouraged to refer patients with spiritual needs to chaplains, support the spiritual/religious beliefs of patients, and pray with patients only if the patient initiated the request. Sharing the provider's own religious beliefs or initiating prayer with patients was not encouraged. Training was most intense during the first month and then tapered off during the rest of the year. A full description of the ETP content is provided elsewhere. ${ }^{18,20}$

Assessments of the program were performed at baseline, 1 month, and 12 months. These times were chosen for the following reasons. Educational efforts and contact with participants were most intense during the first month; this was a time when they were introduced to the program, instructed what to do and why, and encouraged to watch the CME videos. Therefore, most change was expected during the first month. Contact with participants during the rest of the ETP was much less intense; this time was spent primarily reminding participants of what they had learned during the first month and encouraging them to continue to put this into practice. The 12-month follow-up was intended to determine whether training during the first month would produce changes in attitudes and behaviors that would last over the long term (for at least the next 11 months).

\section{Questionnaire}

The questionnaire assessed 1) baseline demographics, practice characteristics, and personal religious characteristics; 2) attitudes toward praying with patients, initiation of prayer, sharing religious beliefs with patients, and encouraging the patients' beliefs (at baseline and each follow-up evaluation); 3) current practices regarding frequency of praying with patients, sharing faith with patients, encouraging patients' own faith, and referrals to chaplains (at baseline and each follow-up); and 4) future willingness to pray with patients and encourage their own religious faith (at baseline and each follow-up). Baseline demographic characteristics included age, gender, race, profession, AHS employee/contract, hospital of primary affiliation, medical specialty, years in practice, religious affiliation (Christian, Jewish, Hindu, Muslim, Buddhist, other religion, none), importance of religion in daily life ("not at all" [1] to "very much" [6]), and exposure during professional training on how to integrate spirituality (none, some, a lot). The rest of the questionnaire administered at each time point focused on the following primary outcomes.

\section{Attitudes}

Concerning prayer with patients, participants were asked: 1) "Should an HP (non-chaplain) ever pray with patients?"; 2) "Should an HP offer to pray with patients?"; and 3) "Should an HP pray with patient if patient initiates request?" ("never" [1] to "always" [6]).

Participants were then asked: 4) "What circumstances should be present for HP to pray with patient?": a) "under no circumstances"; b) "HP must be same religious faith as patient"; c) "HP must have taken a spiritual history"; d) "HP should only say prayer quietly"; e) "HP should only say a prayer aloud"; and f) "only patient, not HP, should say prayer". Next, participants were asked: 5) "When should an HP share their own religious faith with patients?" with the following responses: a) "under no circumstances"; b) "if patient asks"; and c) "whenever HP feels this is appropriate". For questions 4 and 5, "agree" or "disagree" answers were requested for each of the responses listed above; however, many participants responded by circling only the "agree" option because of the response format. If "agree" was circled for any item in the block, then missing responses were designated as "disagree" (14.4\% and $9.0 \%$, at baseline; $15.1 \%$ and $15.6 \%$ at 1 month; $9.3 \%$ and $9.3 \%$ at 12 months, respectively). This procedure was also followed for the question asking about components of ETP completed ("yes" vs "no"; done in $8 \%$ of cases).

Finally, participants were asked: 6) "Should HP encourage patients to become more active in their own religious faith for health reasons?" ("never" [1] to "always" [6]). 


\section{Practices}

Concerning behaviors, providers were asked: 1) "How often do you currently pray with your patients?"; 2) "How often do you currently share your religious faith with patients?"; 3) "How often do you currently encourage patients to become more active in their own religious faith for health reasons?"; and 4) "How often do you currently refer patients to chaplains?" Concerning future behaviors, participants were asked: 5) "Would you be willing to take the time to pray with a patient?"; and 6) "Would you ever consider encouraging patients to become more active in their own religious faith for health reasons?" ("never" to "always" [1-6]). Responses were categorized into low, moderate, and high categories for visual inspection of trends, but were left as continuous variables for statistical analyses.

\section{Statistical analyses}

Frequencies and means with standard deviations were calculated for baseline demographics, religious, and clinical practice characteristics (Table 1), and for participants' responses to questions regarding attitudes/behaviors (Table 2). Growth curve modeling with random intercept and slope (mixedeffects models for continuous outcomes and generalized linear mixed-effects models for binary outcomes) was used to examine changes in attitudes/practices from baseline prior to the initiation of ETP through the end of the 12-month program. An unstructured correlation matrix $(2 \times 2$ dimension) was used for intercepts and slopes for continuous outcomes. This statistical method allowed for participants with data for at least one time point to be included in the analysis and helped to address the problem of missing data. Analyses were conducted for the overall sample to address the primary hypotheses, and were stratified by profession (Table 3). The initial models included time only to assess changes over time during the program. Baseline predictors were then added to models and variables removed where $p>0.15$ in a stepwise fashion until only those with $p<0.10$ remained (Table 4). An interaction term between baseline religiosity and time was added to a model containing time and religiosity to assess the effects of clinician religiosity on outcomes. Effect sizes (Cohen's $d$ ) for changes over time were calculated for the primary hypotheses using degrees of freedom and $t$ values from the mixed models. Alpha level was set at 0.05 and was not adjusted for multiple comparisons because of the exploratory nature of these analyses. All statistical tests were performed using SAS (version 9.3; SAS Institute Inc., Cary, NC, USA).
Table I Baseline characteristics of participants $(n=520)$

\begin{tabular}{ll}
\hline Characteristics & Mean (SD) or \% (n) \\
\hline Age, years, mean (SD) & $44.8(15.5)$ \\
Gender (\% female) & $45.6(234)$ \\
Race (\% White) & $60.5(312)$ \\
Site (\% Florida Hospital) & $66.1(337)$ \\
Specialty (\% family medicine) & $34.7(180)$ \\
Years in practice, mean (SD) & $15.8(11.2)$ \\
Employed/contract with AHS, \% (n) & $59.9(311)$ \\
If AHS employee, years at AHS, mean (SD) & $6.0(7.3)$ \\
Religious affiliation, \% (n) & \\
Christian & $78.7(406)$ \\
Non-Christian & $18.6(96)$ \\
None & $2.7(14)$ \\
Christian denomination, \% (n) & \\
Catholic/Orthodox & $24.1(98)$ \\
Protestant & $33.5(136)$ \\
SDA & $13.8(56)$ \\
Other or missing & $28.6(116)$ \\
Importance of religion, \% (n) & \\
Not at all/slight/some & $16.2(84)$ \\
Moderate or quite a bit & $31.6(164)$ \\
Very much & $52.2(271)$ \\
Exposure during training on how to integrate spirituality, \% (n) & $60.4(312)$ \\
No & $33.5(173)$ \\
Some & $6.2(32)$ \\
A lot &
\end{tabular}

Note: Sample size may vary by up to $1 \%$.

Abbreviations: AHS, Adventist Health Services, SDA, Seventh-Day Adventist; SD, standard deviation.

\section{Results}

Baseline sample characteristics are presented in Table 1. Average age was 45 years, $46 \%$ were women, $61 \%$ White, two-thirds from the Florida Hospital site, one-third family medicine, average time in practice was 16 years, $79 \%$ Christian (if Christian, 14\% Adventist), 52\% indicated religion was very important to them, and $60 \%$ had no exposure to integrating spirituality during their professional training. Of 520 clinicians who completed the baseline questionnaire, 436 (83.8\%) completed the 1-month and $432(83.1 \%)$ completed the 12-month assessments. Reasons for dropping out were insufficient time (33\%), loss of interest (18\%), moved/left practice/retired (18\%), no reason given (19\%), and miscellaneous (12\%). Although no significant differences were found between completers and dropouts on age, gender, race, hospital affiliation, professional training, medical specialty, or years in practice, dropouts were less likely to be AHS employees (50.6\% vs $61.8 \%$ ), less likely to have prior training on integrating spirituality ( $27.3 \%$ vs $42.2 \%)$, less likely to be very religious ( $43.7 \%$ vs $53.9 \%$ ), less likely to be Christian affiliated (73.3\% vs $79.8 \%$ ), and if Christian, more likely to be Catholic/Orthodox (32.8\% vs 22.3\%) and less likely to be Protestant (17.2\% vs $36.7 \%$ ) or Adventist (4.7\% vs $15.9 \%$ ). 
Table 2 Change over 12 months in attitudes/practices regarding spiritual integration

\begin{tabular}{llll}
\hline Attitudes/practices & Baseline & I month & I 2 months \\
& $(n=520)$ & $(n=436)$ & $(n=432)$ \\
& $\%(n)$ & $\%(n)$ & $\%(n)$
\end{tabular}

\section{Should HP pray with patients?}

Yes, sometimes $\quad 54.0(279) \quad 56.1(243) \quad 56.4(242)$

Yes, often or very often $\quad 29.6$ (I53) 29.8 (I29) 31.2 (I34)

Should HP offer to pray with patient?

$\begin{array}{llll}\text { No } & 23.4(121) & 21.0(91) & 20.1(86)\end{array}$

Yes, sometimes $\quad 51.0(264) \quad 51.8(225) \quad 50.5(216)$

Yes, often or very often $\quad 25.7$ (I33) 27.2 (II8) 29.4 (I26)

Should HP pray if patient requests?

$\begin{array}{llll}\text { No } & 6.0(31) & 5.1(22) & 6.1(26)\end{array}$

Yes, sometimes $\quad 21.8$ (II2) 26.0 (II3) 25.3 (108)

Yes, often or very often $\quad 72.2(372) \quad 68.9(299) \quad 68.6(293)$

Circumstances for HP to pray (\% yes)

$\begin{array}{llll}\text { No circumstances exist } & \text { I7.2(88) } & \text { I3.2(56) } & 9.7(4 \mathrm{I})\end{array}$

HP must be same faith as patient 6.1 (3I) $6.6(28) \quad 6.7(28)$

HP must have taken screening $\quad 26.2$ (I33) 26.2 (III) 28.3 (II9)

spiritual history

$\begin{array}{llll}\text { HP should only say } & 14.4(73) & 17.9(76) & 18.8(79)\end{array}$

prayer quietly

HP should only say prayer aloud $\quad 15.0(76) \quad 20.1(85) \quad 19.5(82)$

Only patient, not HP, $\quad 7.9(40) \quad 10.9(46) \quad 6.7(28)$

should pray

Should HP share own faith with patient? (\% yes)

Under no circumstances $\quad 6.8(35) \quad 6.7(29) \quad 5.9(25)$

If patient asks $91.3(473) \quad 88.3(383) \quad 91.1$ (388)

When HP feels it is appropriate 76.8 (398) 76.5 (332) 80.5 (343)

Should HP encourage patient's faith?

$\begin{array}{llll}\text { Never or rarely } & \text { I2.4(64) } & \text { II.4 (49) } & \text { II.8 (5I) }\end{array}$

Sometimes $\quad 38.6$ (199) 35.5 (I53) 34.8 (I50)

Often or always $\quad 48.9(252) \quad 53.1$ (229) $53.4(230)$

Do you pray with patients?

No

Yes, sometimes

$52.7(273) \quad 49.1(213) \quad 41.4(177)$

Yes, often or very often

32.1 (166) $31.6(137) \quad 38.6(165)$

$15.3(79) \quad 19.4(84) \quad 20.1(86)$

Do you share your religious faith with patients?

$\begin{array}{llll}\text { Never or rarely } & 40.8(211) & 39.6(17 I) & 33.6(145)\end{array}$

Sometimes $\quad 35.0$ (18I) 36.3 (I57) 38.3 (165)

Often or always 24.2 (I25) 24.1 (104) 28.1 (I2I)

Do you encourage patient's faith?

$\begin{array}{lllll}\text { Never or rarely } & 32.1(166) & 30.2(131) & 23.1 & (99)\end{array}$

Sometimes $\quad 39.7(205) \quad 34.3(149) \quad 40.8(175)$

Often or always $\quad 28.2$ (146) 35.5 (I54) 36.1 (I55)

Willing to pray with patients?

$\begin{array}{llll}\text { Never or rarely } & \text { I9.7 (I0I) } & \text { I5.9 (69) } & \text { I4.7 (63) }\end{array}$

Sometimes $\quad 46.3(238) \quad 46.7(203) \quad 46.3$ (198)

Often or always $\quad 34.1$ (175) 37.5 (163) 39.0 (167)

Willing to encourage patient's faith?

$\begin{array}{llll}\text { Never or rarely } & \text { I4.2 (73) } & 10.4(45) & \text { II.6 (50) }\end{array}$

Sometimes $\quad 35.9$ (185) 38.5 (167) 36.2 (156)

Often or always $\quad 49.9(257) \quad 51.2(222) \quad 52.2(225)$

How often do you refer patients to chaplains?

Never or rarely $\quad 59.8(309) \quad 54.1$ (233) 46.1 (198)

Sometimes $\quad 28.1(145) \quad 33.6(145) \quad 41.2$ (I77)

Often or always $12.2(63) \quad 12.3(53) \quad 12.8(55)$

Note: Sample size varies by $<2 \%$.

Abbreviation: HP, health professional.
Table 3 Changes over time in attitudes/practices regarding spiritual integration

\begin{tabular}{|c|c|c|}
\hline Attitudes/practices & $\begin{array}{l}\text { Physicians }(n=427) \\
B^{\mathrm{b}}(\mathrm{SE})(d)\end{array}$ & $\begin{array}{l}\text { MLPs }(n=93) \\
B^{b}(S E)(d)\end{array}$ \\
\hline $\begin{array}{l}\text { Should HP pray } \\
\text { with patient? }\end{array}$ & $0.050(0.027)(0.20)^{*}$ & $-0.033(0.053)(-0.14)$ \\
\hline $\begin{array}{l}\text { Should HP offer } \\
\text { to pray? }\end{array}$ & $0.037(0.027)(0.14)$ & $0.017(0.060)(0.07)$ \\
\hline $\begin{array}{l}\text { Should HP pray if } \\
\text { patient initiates? }\end{array}$ & $-0.042(0.025)(-0.18)^{*}$ & $-0.112(0.050)(-0.51)^{\#}$ \\
\hline \multicolumn{3}{|c|}{ No circumstances justify prayer (agree) } \\
\hline I mo (vs baseline) & $-0.438(0.195)(-0.12)^{\#}$ & $-1.034(0.518)(-0.33)^{\#}$ \\
\hline 12 mo (vs baseline) & $-0.777(0.232)(-0.26)^{\$}$ & $-1.004(0.520)(-0.32)^{*}$ \\
\hline \multicolumn{3}{|c|}{ HP should share own faith ${ }^{\mathrm{a}}$ (agree) } \\
\hline I mo (vs baseline) & $0.030(0.177)(0.01)$ & $-0.336(0.406)(-0.14)$ \\
\hline 12 mo (vs baseline) & $0.245(0.184)(0.10)$ & $0.064(0.434)(0.02)$ \\
\hline $\begin{array}{l}\text { Should HP encourage } \\
\text { patient's faith? }\end{array}$ & $0.005(0.032)(0.02)$ & $0.126(0.065)(0.44)^{*}$ \\
\hline $\begin{array}{l}\text { Do you pray with } \\
\text { patients? }\end{array}$ & $0.118(0.022)(0.57)^{\ddagger}$ & $0.173(0.049)(0.80)^{\$}$ \\
\hline $\begin{array}{l}\text { Do you share your } \\
\text { faith with patients? }\end{array}$ & $0.079(0.025)(0.33)^{\ddagger \ddagger}$ & $-0.014(0.050)(-0.07)$ \\
\hline $\begin{array}{l}\text { Do you encourage } \\
\text { patient's faith? }\end{array}$ & $0.102(0.028)(0.38)^{\$}$ & $0.165(0.047)(0.79)^{\$}$ \\
\hline $\begin{array}{l}\text { Willing to pray with } \\
\text { patients? }\end{array}$ & $0.073(0.029)(0.26)^{\ddagger \ddagger}$ & $0.062(0.056)(0.25)$ \\
\hline $\begin{array}{l}\text { Willing to encourage } \\
\text { patient's faith? }\end{array}$ & $0.039(0.028)(0.14)$ & $-0.056(0.059)(-0.21)$ \\
\hline $\begin{array}{l}\text { Do you refer patients } \\
\text { to chaplains? }\end{array}$ & $0.144(0.026)(0.58)^{\ddagger}$ & $0.206(0.062)(0.76)^{\$}$ \\
\hline
\end{tabular}

Notes: ${ }^{*} p<0.10,{ }^{*} p<0.05,{ }^{*} p<0.01,{ }^{*} p<0.001,{ }^{s} p<0.0001$. ${ }^{a} W h e n$ HP feels this is appropriate. ${ }^{b}$ For time $B$ coefficient and $S E$ from mixed-effects regression model; $d$, Cohen's $d$ (effect size, where $0.20=$ small, $0.50=$ medium, $0.80=$ large);

Abbreviations: B, unstandardized beta; HP, health professional; mo, month(s); MLP, mid-level practitioner; SE, standard error; $\mathrm{SSH}$, screening spiritual history.

\section{Change over time} Hypothesis I

Visual inspection suggests that attitude toward praying with patients changed little over time (Table 2), and this was confirmed by results from mixed-effects regression models indicating no significant change over time in the overall sample $(B=0.035$, standard error $[\mathrm{SE}]=0.024$, $p=0.140)$ or in stratified analyses among physicians $(\mathrm{B}=0.050, \mathrm{SE}=0.027, p=0.062)$ or MLPs $(\mathrm{B}=-0.033$, $\mathrm{SE}=0.053, p=0.369$; Table 3). Likewise, visual inspection indicated little change in attitude toward HPs offering to pray with patients, again confirmed by mixed-effects models for the overall sample $(\mathrm{B}=0.033, \mathrm{SE}=0.025$, $p=0.178)$ and in physicians $(\mathrm{B}=0.037, \mathrm{SE}=0.027, p=0.169)$ and MLPs $(\mathrm{B}=0.017, \mathrm{SE}=0.060, p=0.771)$. Surprisingly, however, attitude toward praying when requested by patient actually decreased over time in the overall sample $(\mathrm{B}=-0.054, \mathrm{SE}=0.023, p=0.017)$, especially among MLPs $(\mathrm{B}=-0.112, \mathrm{SE}=0.050, p=0.028)$. 
Table 4 Baseline predictors of change in attitudes/practices regarding spiritual integration

\begin{tabular}{|c|c|c|}
\hline \multirow[t]{2}{*}{ Attitudes/practices } & $\begin{array}{l}\text { Physicians } \\
(n=427)\end{array}$ & $\begin{array}{l}\text { Mid-level practitioners } \\
(n=93)\end{array}$ \\
\hline & B (SE) & B (SE) \\
\hline \multicolumn{3}{|c|}{ Should HP pray with patient? } \\
\hline Time & $0.049(0.027)^{*}$ & $-0.048(0.053)$ \\
\hline Family medicine specialty & $0.209(0.092)^{\#}$ & - \\
\hline Years in practice & $-0.007(0.004)^{\#}$ & - \\
\hline Christian (vs other) & $0.474(0.102)^{\$}$ & $0.706(0.297)^{\#}$ \\
\hline Training during PE & $0.190(0.090)^{\#}$ & - \\
\hline Importance of religion & $0.266(0.031)^{\$}$ & $0.191(0.070)^{\ddagger}$ \\
\hline \multicolumn{3}{|c|}{ Should HP offer to pray? } \\
\hline Time & $0.036(0.027)$ & $0.035(0.036)$ \\
\hline Family medicine specialty & $0.215(0.101)^{\#}$ & - \\
\hline Christian (vs other) & $0.446(0.11 \mathrm{I})^{\$}$ & - \\
\hline Training during PE & $0.275(0.098)^{\ddagger}$ & - \\
\hline Importance of religion & $0.291(0.034)^{\$}$ & $0.363(0.066)^{\$}$ \\
\hline \multicolumn{3}{|c|}{ Should HP pray if patient initiates? } \\
\hline Time & $-0.048(0.025)^{*}$ & $-0.120(0.050)^{\#}$ \\
\hline Race & $0.316(0.084)^{\ddagger \ddagger}$ & - \\
\hline AHS employ/contract & $0.207(0.085)^{\#}$ & - \\
\hline Site (Florida Hospital) & - & $-0.430(0.167)^{\#}$ \\
\hline Training during PE & $0.243(0.087)^{\ddagger}$ & - \\
\hline Importance of religion & $0.297(0.031)^{\$}$ & $0.255(0.061)^{\$}$ \\
\hline \multicolumn{3}{|c|}{ If pray, SSH should be taken (agree) } \\
\hline \multicolumn{3}{|c|}{ Time } \\
\hline I month (vs baseline) & $0.018(0.179)$ & $-0.156(0.373)$ \\
\hline 12 months (vs baseline) & $0.167(0.177)$ & $-0.169(0.379)$ \\
\hline Race & $-0.529(0.187)^{\ddagger}$ & - \\
\hline \multicolumn{3}{|c|}{ HP should share own faith ${ }^{a}$ (agree) } \\
\hline \multicolumn{3}{|c|}{ Time } \\
\hline I month (vs baseline) & $0.020(0.181)$ & $-0.336(0.406)$ \\
\hline I 2 months (vs baseline) & $0.247(0.189)$ & $0.064(0.434)$ \\
\hline Age & $0.013(0.006)^{\#}$ & - \\
\hline Family medicine specialty & $0.619(0.218)^{\ddagger}$ & - \\
\hline Training during $\mathrm{PE}$ & $0.405(0.212)^{*}$ & - \\
\hline Importance of religion & $0.184(0.066)^{\ddagger}$ & - \\
\hline \multicolumn{3}{|c|}{ Should HP encourage patient's faith? } \\
\hline Time & $0.003(0.032)$ & $0.115(0.065)^{*}$ \\
\hline Family medicine specialty & $0.251(0.102)^{\ddagger}$ & - \\
\hline Training during PE & - & $-0.44 \mathrm{I}(0.195)^{\#}$ \\
\hline Importance of religion & $0.333(0.036)^{\$}$ & $0.359(0.075)^{\$}$ \\
\hline \multicolumn{3}{|c|}{ Do you pray with patients? } \\
\hline Time & $0.117(0.021)^{\$}$ & $0.102(0.033)$ \\
\hline Age & $0.006(0.003)^{\#}$ & $0.024(0.007)^{\ddagger \ddagger}$ \\
\hline Race & - & $-0.429(0.242)^{*}$ \\
\hline Christian (vs other) & $0.465(0.106)^{\$}$ & - \\
\hline Importance of religion & $0.326(0.033)^{\$}$ & $0.285(0.075)^{\ddagger \ddagger}$ \\
\hline \multicolumn{3}{|c|}{ Do you share your faith with patients? } \\
\hline Time & $0.076(0.025)^{\ddagger}$ & $-0.025(0.049)$ \\
\hline Age & - & $0.015(0.007)^{\#}$ \\
\hline AHS employ/contract & $-0.157(0.086)^{*}$ & - \\
\hline Christian (vs other) & $0.447(0.103)^{\$}$ & - \\
\hline Training during PE & $0.143(0.086)^{*}$ & - \\
\hline Importance of religion & $0.330(0.032)^{\$}$ & $0.45 \mathrm{I}(0.068)^{\$}$ \\
\hline \multicolumn{3}{|c|}{ Do you encourage patient's faith? } \\
\hline Time & $0.096(0.028)^{\ddagger \ddagger}$ & $0.156(0.047)^{\ddagger}$ \\
\hline Gender & $0.203(0.100)^{\#}$ & - \\
\hline
\end{tabular}

(Continued)
Table 4 (Continued)

\begin{tabular}{|c|c|c|}
\hline \multirow[t]{2}{*}{ Attitudes/practices } & $\begin{array}{l}\text { Physicians } \\
(n=427)\end{array}$ & $\begin{array}{l}\text { Mid-level practitioners } \\
(n=93)\end{array}$ \\
\hline & B (SE) & B (SE) \\
\hline Years in practice & $0.011(0.004)^{\ddagger}$ & - \\
\hline Training during PE & $0.316(0.099)^{\ddagger}$ & $-0.454(0.201)^{\#}$ \\
\hline Importance of religion & $0.241(0.034)^{\$}$ & $0.404(0.076)^{\$}$ \\
\hline \multicolumn{3}{|c|}{ Willing to pray with patients? } \\
\hline Time & $0.066(0.029)^{\#}$ & $0.053(0.057)$ \\
\hline Age & - & $0.018(0.006)^{\ddagger}$ \\
\hline Race & - & $-0.405(0.194)^{\#}$ \\
\hline AHS employ/contract & $0.182(0.091)^{\#}$ & - \\
\hline Christian (vs other) & $0.512(0.109)^{\$}$ & - \\
\hline Training during $\mathrm{PE}$ & $0.198(0.091)^{\#}$ & - \\
\hline Importance of religion & $0.357(0.034)^{\$}$ & $0.342(0.062)^{\$}$ \\
\hline \multicolumn{3}{|c|}{ Willing to encourage patient's faith? } \\
\hline Time & $0.034(0.028)$ & $-0.063(0.059)$ \\
\hline Age & $0.007(0.003)^{\#}$ & $0.013(0.007)^{*}$ \\
\hline Gender & $0.260(0.097)^{\ddagger}$ & - \\
\hline Family medicine specialty & $0.235(0.095)^{\ddagger}$ & - \\
\hline Training in PE & $0.151(0.058)^{\ddagger}$ & $-0.35(0.196)^{*}$ \\
\hline Importance of religion & $0.272(0.033)^{\$}$ & $0.291(0.076)^{\ddagger \ddagger}$ \\
\hline \multicolumn{3}{|c|}{ How often refer patients to chaplains? } \\
\hline Time & $0.142(0.026)^{\$}$ & $0.204(0.062)^{\ddagger}$ \\
\hline Age & - & $0.018(0.007)^{\ddagger}$ \\
\hline Training in $\mathrm{PE}$ & $0.213(0.080)^{\ddagger}$ & - \\
\hline
\end{tabular}

Notes: $\mathrm{N}$ varies depending on covariates in model, "-" indicates no association $(p \geq 0.10),{ }^{*} p<0.10,{ }^{*} p<0.05,{ }^{\ddagger} p<0.01,{ }^{* \neq} p<0.001,{ }^{\$} p<0.0001$. ${ }^{a}$ When HP feels this is appropriate.

Abbreviations: AHS, Adventist Health System; B, unstandardized beta; HP, health professional; SSH, screening spiritual history; SE, standard error; PE, professional education.

With regard to circumstances that should be present for HP to pray with a patient, visual inspection suggests that the largest change occurred in the category that no circumstances existed that would justify praying with patients, decreasing from $17.2 \%$ at baseline to $13.2 \%$ at 1 -month and $9.7 \%$ at the 12 -month follow-up. Results from mixed-effects regression models confirmed this change for the overall sample (for baseline to 1-month follow-up, $\mathrm{B}=-0.438, \mathrm{SE}=0.195, p=0.025$; for baseline to 12-month follow-up, $\mathrm{B}=-0.812, \mathrm{SE}=0.212, p=0.0001$ ), and in both physicians and MLPs in stratified analyses, especially from baseline to 12-month follow-up in physicians (Table 3).

There was no change in attitudes toward when HPs should share their own religious faith with patients, other than nearly unanimous agreement across time (88\%-91\%) that this was appropriate to do if the patient asks. Few felt that under no circumstances should HPs share their faith (as the ETP stressed) and a clear majority (77\%-81\%) felt that it was appropriate to do so based on the clinician's judgment. No significant change during the program occurred in the attitude toward sharing HPs' religious faith (when felt appropriate) either for the overall sample (baseline to 1 month $\mathrm{B}=-0.028, \mathrm{SE}=0.162, p=0.862$; baseline to 12 months 
$\mathrm{B}=0.215, \mathrm{SE}=0.169, p=0.204$ ) or for physicians or MLPs when examined separately (Table 3 ).

Visual inspection also suggested a small increase over time in the view that HPs should often or always encourage the patient's own religious faith, increasing from $48.9 \%$ at baseline to $53.4 \%$ at 12 -month follow-up. However, this did not reach statistical significance in the overall sample $(\mathrm{B}=0.026, \mathrm{SE}=0.028, p=0.364)$ or in analyses stratified by profession, although approached significance in MLPs $(\mathrm{B}=0.126, \mathrm{SE}=0.065, p=0.081)$.

\section{Hypothesis 2}

In contrast to attitudes that changed relatively little overall, behaviors changed more substantially. This was true for frequency of praying with patients, with the proportion of those doing so (at least sometimes) modestly increasing from $47.4 \%$ at baseline to $58.7 \%$ at 12 -month follow-up, a significant change over time in the overall sample $(\mathrm{B}=0.128$, $\mathrm{SE}=0.020, p<0.0001$, Figure 1), and in both physicians and MLPs (Table 3). Likewise, frequency of chaplain referral also increased from $40.3 \%$ to $54.0 \%$, a trajectory that was significant across time for the overall sample $(\mathrm{B}=0.155, \mathrm{SE}=0.024$, $p<0.0001)$ and in both physicians and MLPs.

Surprisingly, frequency of clinicians sharing their religious faith with patients increased over time in the overall sample $(\mathrm{B}=0.063, \mathrm{SE}=0.022, p=0.005)$, especially in physicians $(\mathrm{B}=0.079, \mathrm{SE}=0.025, p=0.002)$. The practice of encouraging the patient's religious faith, though, also increased over time in the overall sample $(\mathrm{B}=0.112, \mathrm{SE}=0.024, p<0.0001$, Figure 2) and in both professions.

Finally, willingness to pray with patients increased over time in the overall sample $(\mathrm{B}=0.072, \mathrm{SE}=0.026, p=0.006)$,

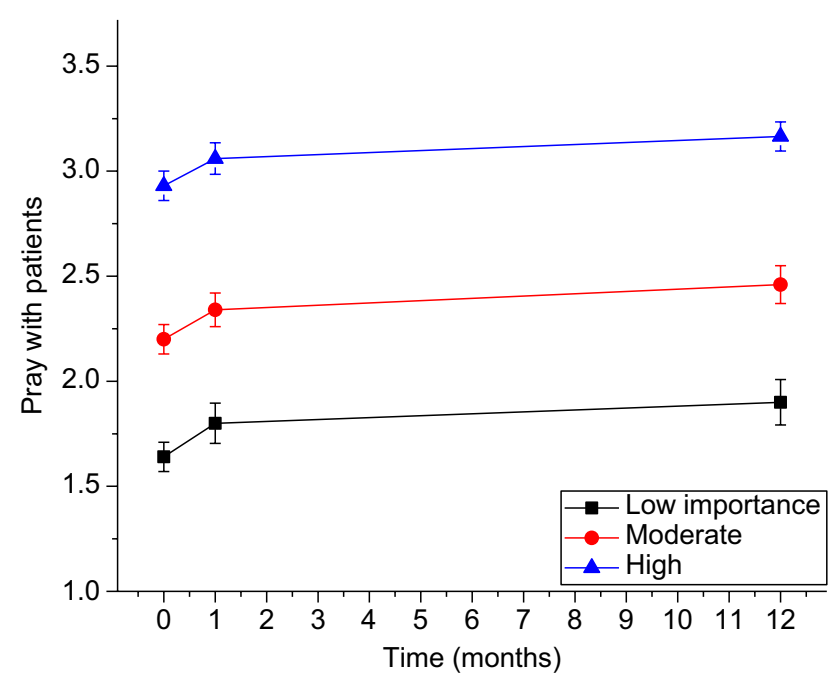

Figure I Changes over time in prayer with patients by importance of religion in the clinician's life. especially in physicians $(\mathrm{B}=0.073, \mathrm{SE}=0.029, p=0.012)$. Willingness to encourage the patient's own religious faith, however, did not increase over time in either physicians or MLPs, despite emphasis in the training program. Not surprisingly, those showing unwillingness to do so were primarily clinicians who at the 12-month follow-up reported that they seldom supported their patients' faith, whereas among clinicians who often or always did so, there was an increase in willingness over time to continue to do so $(\mathrm{B}=0.156$, $\mathrm{SE}=0.040, p<0.0001)$.

\section{Hypothesis 3}

Table 4 describes the characteristics of clinicians that predicted an increased likelihood of positive attitudes/behaviors toward engaging in spiritual practices with patients from baseline through the 12-month follow-up. With regard to attitudes, physicians who had received exposure to integrating spirituality into patient care during medical school or were family medicine specialists were especially likely to believe that HPs should pray with patients, offer prayer to patients, share their own faith with patients, and encourage the patient's faith. With regard to actual behavior across time, older clinicians were more likely to pray with patients (both MDs and MLPs), more willing to pray with patients (MLPs), more likely to share their faith (MLPs), more willing to encourage the patient's faith (MDs), and more likely to make chaplain referrals (MLPs). Female physicians were more likely across time to encourage the patient's own religious faith and be willing to do so in the future. Christian physicians were more likely to pray with patients, be willing to pray with patients, and share their faith with patients. Physicians employed by AHS were more willing to pray with patients, but tended to be less likely to share

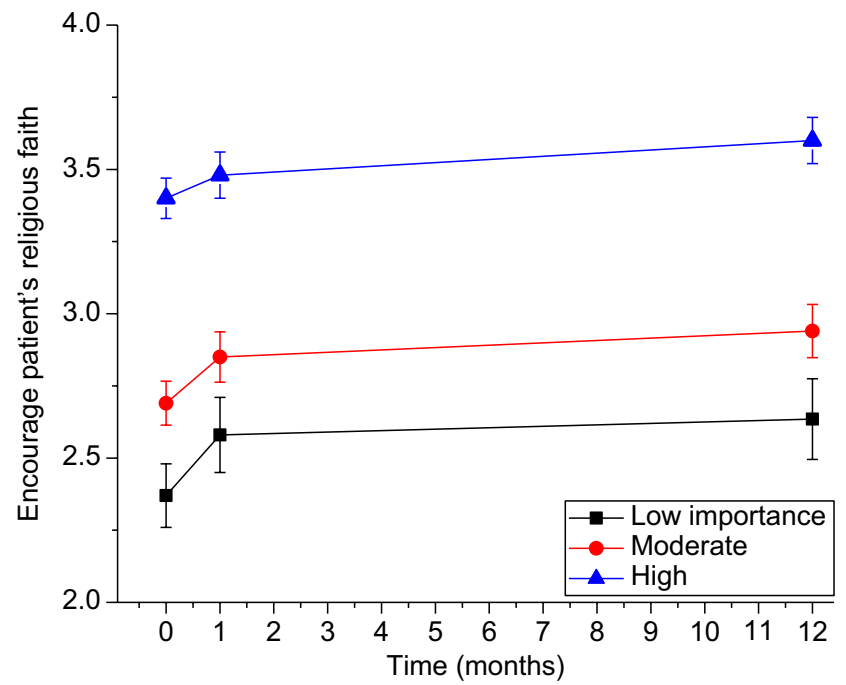

Figure 2 Changes over time in encouragement of patient's faith by importance of religion in the clinician's life. 
their faith with patients. White MLPs (vs non-White) were less likely to pray with patients or be willing to pray with patients.

Importance of religion in the clinician's daily life, however, was the strongest predictor of positive attitudes and behaviors in both physicians and MLPs across time. This was true for prayer with patients, sharing faith with patients, and encouraging the patient's own religious faith (although had little influence on frequency of referral to chaplains).

\section{Hypothesis 4}

No significant interaction was found between religiosity and time in the model examining behaviors concerning prayer with patients, sharing faith with patients, encouraging the patient's own religious faith, or referral to chaplains, suggesting (contrary to our hypothesis) that the intervention effectively increased these behaviors in both religious and non-religious clinicians.

\section{Discussion}

To our knowledge, this is the first attempt by a health care system to comprehensively train clinicians on how to sensitively and appropriately engage in spiritual practices with patients in an outpatient setting. In addition to examining the effects of the training program on frequency of chaplain referral, a common and accepted practice, this report also examines what effects the ETP had on controversial spiritual practices with patients, some of which may involve boundary violations. For example, some experts have discouraged clinicians from praying with patients, indicating that only trained chaplains or clergy should do so. ${ }^{21-23}$ The ethical concern is that if clinicians conduct an activity with patients that goes beyond their scope of practice and that has not been requested by the patient, this may result in the "appearance of religious coercion", that is, clinicians engaging in a practice that they are not trained for and that patients are not expecting or wanting. ${ }^{23}$ That concern increases when clinicians share their own religious beliefs with patients, even if they feel that it is appropriate (although is lessened if the patient asks about the clinician's beliefs). Proselytizing has been almost universally condemned because of the unequal power status between the clinician and the patient. ${ }^{21-24}$ Religious or spiritual belief is a sensitive, though important, area of patients' lives and is related to their health and medical care. However, given the pluralistic nature of health care settings, most agree that when spiritual issues related to health are addressed in clinical care, they should be centered on the beliefs of the patient (patient-centered) and not on those of the clinician (clinician-centered). The clinician's religiosity or particular religion should not matter; it is the patient's beliefs and understanding of their spiritual needs that should be central.

However, clinicians who are more religious may be more open to addressing spiritual issues that patients struggle with, and because of their interest in the area, may be more aware of the research showing connections with health and the rationale for integrating spirituality into patient care. Religious clinicians, then, may value this topic sufficiently to take the necessary time to engage in spiritual practices with patients if requested. With regard to prayer, many patients rely on this practice when dealing with health issues. A national random sample of 31,044 US adults found that "prayer for health reasons" was the most common (45.2\% in the past 12 months) of all types of complementary and alternative medicine therapies that were engaged in, ${ }^{25}$ especially by Hispanics $(49.5 \%)$ and African-Americans $(62.6 \%)^{26}$. Furthermore, many patients indicate favorable attitudes toward prayer with their medical provider, ranging from $19 \%$ to $95 \%,{ }^{27-32}$ and nearly two-thirds of the Americans believe that physicians should pray with patients if the patient asks. ${ }^{33}$

Besides our initial report on the baseline prevalence of attitudes/practices of clinicians with regard to prayer with patients, sharing their faith, and encouraging patients' own religious beliefs, ${ }^{34}$ there is not much systematic information on these practices among outpatient providers. In a random sample of 160 family physicians in Illinois, conducted over 30 years ago, participants were asked if they had ever prayed with an older patient and, if so, whether they thought this had helped. Researchers found that $37 \%$ had prayed with a patient, $89 \%$ indicated that it had helped, and $88 \%$ said that it was appropriate to pray with a sick older patient if the patient requested. ${ }^{35}$ However, Monroe et al in a survey of 476 physicians at academic medical centers in Florida, North Carolina, and Vermont (the majority of whom were internists) found that only $6 \%$ said that physicians should initiate prayer with a patient during a routine office visit. ${ }^{36}$ Likewise, in a study of 108 Northeastern US physicians, only $7 \%$ said that initiating prayer with patients was appropriate (and 61\% frankly disagreed with this practice). ${ }^{37}$ In the only random national sample of US physicians $(n=1,144), 81 \%$ never or rarely prayed with patients and $59 \%$ said that they never or rarely share their own religious beliefs. ${ }^{10}$

The intervention (ETP) used in the present study sought to educate, train, and support clinicians in sensitive and appropriate ways of integrating spirituality into patient care. The program had surprisingly little effect over time on changing clinicians' attitudes toward praying with patients, sharing their faith, or supporting patients' own religious faith. 
Instead, the training program affected clinician behaviors more than their attitudes. Frequency of clinician-patient prayer increased, sharing their personal faith increased (surprisingly, and despite the training that tended to discourage this practice), encouraging the patient's own faith increased, and referrals to chaplains increased (as did taking a spiritual history ${ }^{18}$ ). While the ETP program was designed to change both attitudes and behaviors, the personal encouragement and support provided throughout the program by RFCs focused on changing behavior (asking providers if they were assessing and addressing patients' spiritual needs), which would explain why behavior changed more than attitude. Although attitudes usually drive practice, the aim of the ETP was to provide clinicians first with the knowledge base and then with the experience of engaging in spiritual practices with patients, hoping that positive experiences plus greater knowledge would ultimately change their attitude (and sustain the practice).

As hypothesized, certain characteristics distinguished clinicians more likely to report positive attitudes to and behaviors regarding engagement in spiritual activities with patients across time. Those were providers who had exposure to integrating spirituality in their professional training (at least for physicians), or were family medicine specialists (providers having a long-term relationship with patients and perhaps more attuned to psychosocial issues), confirming reports from cross-sectional studies. ${ }^{10,38}$ They also tended to be older. This may have reflected greater experience/confidence in addressing these issues, or could indicate a shift toward a more secular society reflected in the attitudes and behaviors of younger clinicians. In particular, though, clinicians who were more religious (as discussed above) showed positive attitudes and behaviors that were sustained over time, again as other studies have found..$^{10,38,39}$ However, contrary to our hypothesis, there was no significant interaction between baseline provider religiosity and time, indicating that the changes observed were not limited to highly religious clinicians only, but also applied to those who were less religious or not religious at all. Thus, this particular training intervention had a similar impact across varying levels of clinician religiosity, which would argue for its effectiveness in facilitating the assessment and addressing of spiritual needs in other health systems made up of providers who are less religious than that found in the AHS.

Finally, changes over time in attitudes and practices were fairly similar in physicians and MLPs. Although significance levels differed in several instances (because of the larger number of physicians), the actual size of the effect (B) varied little in most cases. There was one notable exception, however. While physicians were significantly more likely over time to share their own personal religious beliefs with patients, this practice did not change in MLPs (who were actually somewhat less likely to do so over time). Because the ETP tended to discourage providers from sharing beliefs because of the appearance of coercion or proselytizing, the MLPs apparently got this message more clearly than did the physicians.

Future research is needed to replicate and generalize these findings. While these results can only be applied to outpatient practices, similar research is needed in inpatient settings where the need to address spiritual issues is even greater given the more severe illness and the increase in spiritual needs during hospitalization because of that. Furthermore, future research should consider surveying patients in addition to providers, before and after the training intervention, to determine if patients can detect any change in the clinicians' behavior as a consequence of the educational program that positively impacts the quality of spiritual care they are receiving.

\section{Limitations}

First and foremost, since there was no control group, is that changes identified here were simply due to the passage of time, repeated assessments and monitoring, and had nothing to do with the ETP intervention or its contents. A randomized trial design would be necessary to rule out this possibility.

Secondly, participants were volunteers and could not be forced to participate in all educational activities and resources; as a result, participation was variable in extent (although such variable engagement is typical for "real-life" settings). Participants were affiliated with the AHS and were largely from the Southeastern USA. Dropouts were less likely to be Christian, Protestant, and less religious than those who completed the study. Power issues may also have influenced results reported for MLPs. All these factors may limit generalizability of the findings. Strictly speaking, then, these results are primarily applicable to the AHS and probably other faith-based health systems. However, $<15 \%$ of the sample was Adventist, one-third of the sample was Protestant Christians, and a quarter was Catholics/Orthodox, which is not greatly different than the distribution of providers in the US population ${ }^{10}$ (except for more Adventists).

Finally, no attempt was made to define "spirituality" for participants, leaving open the possibility that clinicians interpreted this term differently, which may have affected response to the intervention. 
Despite these limitations, the strengths of the study are its diverse and relatively large sample of clinicians from several areas of the USA, the high response rate at both follow-ups (over $80 \%$ ), and use of the latest statistical methods for analyzing trajectories of change over time and their predictors. As mentioned earlier, this is the first time to our knowledge that an entire health care system has attempted to change the beliefs and behaviors of clinicians related to integrating spirituality into outpatient clinical care.

\section{Conclusion}

The ETP intervention administered here, while largely ineffective in changing clinician beliefs or attitudes, did appear to affect the frequency of several often controversial practices involved in integrating spirituality into medical care, including clinician-patient prayer, sharing clinicians' faith with patients, encouraging the patients' own religious faith for health reasons, and referring patients to chaplains, with the initial increases sustained over a 12-month follow-up period. While clinician religiosity was a strong predictor of these behaviors across time, no evidence was found that the intervention was effective only in religious clinicians. Rather, the increase in these practices was independent of clinician religiosity. This leaves open the possibility that training interventions of this kind may be useful for increasing wholeperson care that attends to the spiritual needs of patients not only in faith-based health systems but also in secular systems (perhaps with a greater emphasis on clinicians praying with patients only if requested and sharing personal religious beliefs only if asked).

Although the optimal time for introduction and vertical integration of training in spiritual care is during medical school (which it is now increasingly but inconsistently done $^{40}$ ), the training of clinicians who are already in practice on how to assess and address spiritual needs, or their retraining, should be made available to all providers as part of CME/ CE programs. Such a program, the same one used to provide the evidence base for participants in the current study, is now available online for free or for $\mathrm{CME} / \mathrm{CE}$ credit. ${ }^{18}$

\section{Disclosure}

The authors report no conflicts of interest in this work.

\section{References}

1. VanderWeele TJ, Li S, Tsai AC, Kawachi I. Association between religious service attendance and lower suicide rates among US women. JAMA Psychiatry. 2016;1:73(8):845-851.

2. Li S, Okereke OI, Chang SC, Kawachi I, VanderWeele TJ. Religious service attendance and lower depression among women - a prospective cohort study. Ann Behav Med. 2016;50(6):876-884.
3. Li S, Stampfer MJ, Williams DR, VanderWeele TJ. Association of religious service attendance with mortality among women. JAMA Intern Med. 2016;176(6):777-785.

4. Ironson G, Kremer H, Lucette A. Relationship between spiritual coping and survival in patients with HIV. J Gen Intern Med. 2016;31(9): 1068-1076.

5. Koenig HG, King DE, Carson VB. Handbook of Religion and Health. 2nd ed. New York, NY: Oxford University Press; 2012.

6. Pearce MJ, Coan AD, Herndon JE 2nd, Koenig HG, Abernethy AP. Unmet spiritual care needs impact quality of life and well-being in advanced cancer. Support Care Cancer. 2012;20(10):2269-2276.

7. Büssing A, Janko A, Baumann K, Hvidt NC, Kopf A. Spiritual needs among patients with chronic pain diseases and cancer living in a secular society. Pain Med. 2013;14(9):1362-1373.

8. Balboni T, Balboni M, Paulk ME, et al. Support of cancer patients' spiritual needs and associations with medical care costs at the end of life. Cancer. 2011;117(23):5383-5391.

9. Joint Commission for the Accreditation of Hospital Organizations (JCAHO) Requirements, RI.01.01.01, EP6 (hospital and ambulatory versions). The Joint Commission, Edition ${ }^{\circledR}$. Oak Brook, IL: Joint Commission Resources; 2016.

10. Curlin FA, Chin MH, Sellergren SA, Roach CJ, Lantos JD. The association of physicians' religious characteristics with their attitudes and self-reported behaviors regarding religion and spirituality in the clinical encounter. Med Care. 2006;44(5):446-453.

11. National Organization of Nurse Practitioner Faculties. 2012 nurse practitioner core competencies (current): independent practice competencies, 4C, p 5; 2016. Available from: http://www.nonpf.org/?page=14. Accessed April 24, 2016.

12. Berg GM, Whitney MP, Wentling CJ, Hervey AM, Nyberg S. Physician assistant program education on spirituality and religion in patient encounters. J Physician Assist Educ. 2013;24(2):24-27.

13. Peteet JR, Balboni MJ. Spirituality and religion in oncology. CA Cancer J Clin. 2013;63(4):280-289.

14. Cobb M, Puchalski CM, Rumbold B. Oxford Textbook of Spirituality in Healthcare. New York, NY: Oxford University Press; 2012.

15. Puchalski CM, Kheirbek R, Doucette A, Martin T, Yang YT. Spirituality as an essential element of person-centered compassionate care: a pilot training program for clinicians. J Med Person. 2013;11(2):56-61.

16. Balboni MJ, Sullivan A, Enzinger AC, et al. Nurse and physician barriers to spiritual care provision at the end of life. J Pain Symptom Manage. 2014;48(3):400-410.

17. Marshall E (2015). 10 largest nonprofit hospital systems. Hospital review; 2015. Available from: http://www.beckershospitalreview.com/ lists/10-largest-nonprofit-hospital-systems-2015.html. Accessed April $16,2016$.

18. CME videos. Spirituality in patient care. Durham, NC: Duke University Center for Spirituality, Theology and Health; 2014. Available from: http://www.spiritualityandhealth.duke.edu/index.php/cme-videos. Accessed August 8, 2016.

19. Koenig HG, Perno K, Erkanli A, Hamilton T. Effects of a 12-month educational program on outpatient clinicians' attitudes and practices concerning the screening spiritual history. South Med J. In press 2017.

20. Koenig HG. The spiritual care team: enabling the practice of whole person medicine. Religions. 2014;5(4):1161-1174.

21. Sloan RP, Bagiella E, VandeCreek L, et al. Should physicians prescribe religious activities? N Engl J Med. 2000;342:1913-1916.

22. Post SG, Puchalski C, Larson DB. Physicians and patient spirituality: professional boundaries, competency, and ethics. Ann Intern Med. 2000;132:578-583.

23. Dagi TF. Prayer, piety, and professional propriety: limits on religious expression on hospitals. J Clin Ethics. 1995:6(3):274-279.

24. Koenig HG. Spirituality in Patient Care. 3rd ed. Philadelphia, PA: Templeton press; 2013.

25. Barnes PM, Powell-Griner E, McFann K, Nahin RL. Complementary and alternative medicine rise among adults: United States, 2002. Advance Data. 2004;343:1-20. 
26. Gillum F, Griffith DM. Prayer and spiritual practices for health reasons among American adults: the role of race and ethnicity. J Relig Health. 2010;49(3):283-295.

27. MacLean CD, Susi B, Phifer N, et al. Patient preference for physician discussion and practice of spirituality. J Gen Intern Med. 2003;18(1):38-43.

28. King DE, Bushwick B. Beliefs and attitudes of hospital inpatients about faith healing and prayer. J Fam Pract. 1994;39(4):349-352.

29. Kaldjian LC, Jekel JF, Friedland G. End-of-life decisions in HIV-positive patients: the role of spiritual beliefs. AIDS. 1998;12(1):103-107.

30. Oyama O, Koenig HG. Religious beliefs and practices in family medicine. Arch Fam Med. 1998;7(5):431-435.

31. McCord G, Gilchrist VJ, Grossman SD, et al. Discussing spirituality with patients: a rational and ethical approach. Ann Fam Med. 2004;2(4): 356-361.

32. Siatkowski RM, Cannon SL, Farris BK. Patients' perception of physician-initiated prayer prior to elective ophthalmologic surgery. South Med J. 2008;101(2):138-141.

33. Yankelovich Parners, Inc. Faith \& healing: can prayer, faith, and spirituality really improve your physical health? a growing and surprising body of scientific evidence says they can. Time Magazine. 1996;147(26): $58-68$.
34. Koenig HG, Perno K, Hamilton T. Integrating spirituality into outpatient practice in the Adventist Health System. South Med J. 2017;110(1): $1-7$.

35. Koenig HG, Bearon L, Dayringer R. Physician perspectives on the role of religion in the physician-older patient relationship. J Fam Pract. 1989; 28:441-448.

36. Monroe MH, Bynum D, Susi B, et al. Primary care physician preferences regarding spiritual behavior in medical practice. Arch Intern Med. 2003;163(22):2751-2756.

37. King SD, Dimmers MA, Langer S, Murphy PE. Doctors' attentiveness to the spirituality/religion of their patients in pediatric and oncology settings in the Northwest USA. J Health Care Chaplain. 2013;19(4): 140-164.

38. Best M, Butow P, Olver I. Doctors discussing religion and spirituality: a systematic literature review. Palliat Med. 2016;30(4):327-337.

39. Balboni MJ, Sullivan A, Amobi A, et al. Why is spiritual care infrequent at the end of life? Spiritual care perceptions among patients, nurses, and physicians and the role of training. J Clin Oncol. 2013;31(4):461-467.

40. Koenig HG, Hooten EG, Lindsay-Calkins E, Meador KG. Spirituality in medical school curricula: findings from a national survey. Int $J$ Psychiatry Med. 2010;40(4):391-398.
Advances in Medical Education and Practice

\section{Publish your work in this journal}

Advances in Medical Education and Practice is an international, peerreviewed, open access journal that aims to present and publish research on Medical Education covering medical, dental, nursing and allied health care professional education. The journal covers undergraduate education, postgraduate training and continuing medical education

\section{Dovepress}

including emerging trends and innovative models linking education, research, and health care services. The manuscript management system is completely online and includes a very quick and fair peer-review system. Visit http://www.dovepress.com/testimonials.php to read real quotes from published authors.

Submit your manuscript here: http://www.dovepress.com/advances-in-medical-education-and-practice-journal 